\title{
The Global Solutions and Moment Boundedness of Stochastic Multipantograph Equations
}

\author{
Maosheng Tian, ${ }^{1}$ Xuejing Meng, ${ }^{2,3}$ Jihong Chen, ${ }^{1}$ and Xiaoqi Tang ${ }^{1}$ \\ ${ }^{1}$ State Engineering Research Center of Numerical Control System, Huazhong University of Science and Technology, \\ Wuhan 430074, China \\ ${ }^{2}$ Postdoctoral Research Station of Zhongnan University of Economics and Law, Wuhan 430073, China \\ ${ }^{3}$ School of Statistics, Hubei University of Economics, Wuhan 430205, China
}

Correspondence should be addressed to Xuejing Meng; mengxuejing@yeah.net

Received 7 August 2015; Accepted 28 January 2016

Academic Editor: Petko Petkov

Copyright (C) 2016 Maosheng Tian et al. This is an open access article distributed under the Creative Commons Attribution License, which permits unrestricted use, distribution, and reproduction in any medium, provided the original work is properly cited.

We consider the existence of global solutions and their moment boundedness for stochastic multipantograph equations. By the idea of Lyapunov function, we impose some polynomial growth conditions on the coefficients of the equation which enables us to study the boundedness more applicably. Methods and techniques developed here have the potential to be applied in other unbounded delay stochastic differential equations.

\section{Introduction}

Delay differential equations (DDEs) play an important role in applied mathematics owing to providing a powerful model of many phenomena, such as some physical applications with noninstant transmission phenomena, neural networks, or other memory processes, and specially biological motivations (e.g., [1-3]) like species' growth or incubating time on disease models among many others.

An interesting case of DDEs which is the subject of a lot of papers is the pantograph equation:

$$
\begin{aligned}
& \dot{x}(t)=f(t, x(t), x(q t)), \quad t \geq 0 \\
& x(0)=x_{0},
\end{aligned}
$$

where $0<q \leq 1, x_{0} \in \mathbb{R}^{n}$. The name pantograph originated from the work of Ockendon and Tayler [4] on the collection of current by the pantograph head of an electric locomotive. The pantograph equations appeared in modeling of various problems such as number theory, astrophysics, nonlinear dynamical systems, biology, economy, quantum mechanics, and electrodynamics. For some applications of this type of equations, we refer to [4-8].

Since any realistic systems are inevitably subject to environmental noise, the stochastic pantograph equation

$$
\begin{aligned}
d x(t)= & f(t, x(t), x(q t)) d t \\
& +g(t, x(t), x(q t)) d w(t), \quad t \geq 0, \\
x(0)= & x_{0}
\end{aligned}
$$

therefore receives more and more attention. Fan et al. [9] have given the sufficient conditions of existence and uniqueness of the solutions and convergence of semi-implicit Euler methods for (2). Appleby and Buckwar [10] have studied the asymptotic growth and decay properties of solutions of the linear stochastic pantograph equation with multiplicative noise. For more literatures we refer the interested reader to [11-13].

Properties of the analytic solution of (1) and (2) as well as numerical methods have been studied by several authors, for example, Lü and Cui [14], Iserles [15, 16], Liu et al. [17], and 
Appleby and Buckwar [10]. A more general form than (1) is the multipantograph equation; it reads

$$
\begin{aligned}
& \dot{x}(t)=f\left(t, x(t), x\left(q_{1} t\right), x\left(q_{2} t\right), \ldots, x\left(q_{L} t\right)\right), \\
& x(0)=x_{0},
\end{aligned}
$$

where $0<q_{1}<q_{2}<\cdots<q_{L}<1$. Equation (3) was also studied by many authors numerically and analytically (see, e.g., [18-20] and the references cited therein).

However, to the best of our knowledge, there are no corresponding numerical and analytical results on stochastic multipantograph equations which also have numerous applications as (2) in engineering and science. It has the form

$$
\begin{aligned}
d x(t)= & f\left(t, x(t), x\left(q_{1} t\right), \ldots, x\left(q_{L} t\right)\right) d t \\
& +g\left(t, x(t), x\left(q_{1} t\right), \ldots, x\left(q_{L} t\right)\right) d w(t), \\
x(0)= & x_{0}, \quad t \geq 0,
\end{aligned}
$$

where $0<q_{1}<q_{2}<\cdots<q_{L}<1, f: \mathbb{R}_{+} \times \mathbb{R}^{n(L+1)} \rightarrow$ $\mathbb{R}^{n}$, and $g: \mathbb{R}_{+} \times \mathbb{R}^{n(L+1)} \rightarrow \mathbb{R}^{n \times m}$. In this paper, we mainly study the asymptotic properties of the analytic solution of (4). Owing to the fact that the delay is unbounded, many methods which are useful for the bounded delay systems are inefficient or impossible for these systems. For example, some classical techniques such as Lyapunov direct methods in [21-23] cannot be transferred directly to the study of boundedness properties for unbounded delay equation (4). By introducing a decay function to control the unbounded delay term, we develop the traditional techniques like Lyapunov direct methods to be applied in the pantograph equations' cases.

It is well known for stochastic differential equations that the linear growth condition plays an important role in suppressing the potential explosion of solutions and guarantees the existence of the global solutions (cf. [22-25]). This paper, without the linear growth condition, shows that (4) almost surely makes a global solution $x\left(t, x_{0}\right)$ and this solution is bounded in the sense

$$
\begin{array}{r}
\limsup _{t \rightarrow \infty} \mathbb{E}\left|x\left(t, x_{0}\right)\right|^{p} \leq K_{p}, \\
\limsup _{t \rightarrow \infty} \frac{1}{t} \int_{0}^{t} \mathbb{E}\left|x\left(s, x_{0}\right)\right|^{q} d s \leq L_{q},
\end{array}
$$

where $p, q, K_{p}$, and $L_{q}$ are positive constants independent of the initial data $x_{0}$.

The content of the paper is as follows. In Section 2, we give some necessary notations and useful lemmas. Section 3 is devoted to presenting a general theorem for the existence and boundedness of the global solution. In Section 4, we apply Theorem 4 to obtain two useful criteria which can be easily verifiable in applications. Two examples are provided to show how our results will be applied in Section 5. Further remarks are made to conclude the paper in the final section.

\section{Some Preliminaries}

Throughout this paper, unless otherwise specified, we use the following notations. Let $(\Omega, \mathscr{F}, \mathbb{P})$ be a complete probability space with a filtration $\left\{\mathscr{F}_{t}\right\}_{t \geq 0}$ satisfying the usual conditions; that is, it is right continuous and increasing while $\mathscr{F}_{0}$ contains all $\mathbb{P}$-null sets. Let $w(t)$ be an $m$-dimensional Brownian motion defined on the probability space. Let $|\cdot|$ be the Euclidean norm in $\mathbb{R}^{n}$. If $A$ is a vector or matrix, its transpose is denoted by $A^{T}$; if $A$ is a matrix, its trace norm is denoted by $|A|=\sqrt{\operatorname{trace}\left(A^{T} A\right)}$. Let $\mathbb{R}_{+}=[0, \infty) ; \mathbb{R}_{\times}=\mathbb{R} \backslash\{0\}$ and $\mathbb{R}_{\times}^{n}=\mathbb{R}^{n} \backslash\{0\}$.

Moreover, let $C^{2}\left(\mathbb{R}^{n} ; \mathbb{R}_{+}\right)$denote the family of all functions $V(x)$ from $\mathbb{R}^{n}$ to $\mathbb{R}_{+}$which are continuously twice differentiable. For all $t \geq 0, x \in \mathbb{R}^{n}$, and $Y \in \mathbb{R}^{n \times L}$ and any $V(x) \in C^{2}\left(\mathbb{R}^{n} ; \mathbb{R}_{+}\right)$, define a function $\mathscr{L} V: \mathbb{R}_{+} \times \mathbb{R}^{n} \times \mathbb{R}^{n \times L} \rightarrow$ $\mathbb{R}$ by

$$
\begin{aligned}
\mathscr{L} V(t, x, Y) \\
=V_{x}(x) f(t, x, Y) \\
\quad+\frac{1}{2} \operatorname{trace}\left[g^{T}(t, x, Y) V_{x x}(x) g(t, x, Y)\right],
\end{aligned}
$$

where $Y=\left(y_{1}, y_{2}, \ldots, y_{L}\right) \in \mathbb{R}^{n \times L}$,

$$
\begin{aligned}
V_{x}(x) & =\left(\frac{\partial V(x)}{\partial x_{1}}, \frac{\partial V(x)}{\partial x_{2}}, \ldots, \frac{\partial V(x)}{\partial x_{n}}\right), \\
V_{x x}(x) & =\left[\frac{\partial^{2} V(x)}{\partial x_{i} \partial x_{j}}\right]_{n \times n} .
\end{aligned}
$$

Thus, if $x(t)$ is a solution of (4), by Itô's formula, we have

$$
\begin{aligned}
d V(x(t))= & L V(x(t)) d t \\
& +V_{x}(x(t)) g(t, x(t), Y(t)) d w(t),
\end{aligned}
$$

where

$$
\begin{aligned}
L V(x(t)) & =\mathscr{L} V(t, x(t), Y(t)), \\
Y(t) & =\left(y_{1}(t), y_{2}(t), \ldots, y_{L}(t)\right), \\
y_{i}(t) & =x\left(q_{i} t\right), \quad i=1, \ldots, L .
\end{aligned}
$$

For coefficients $f$ and $g$, we will impose the following standing assumptions.

Assumption 1. Both $f$ and $g$ satisfy the local Lipschitz condition. That is, for each $j=1,2, \ldots$, there exists a constant $c_{j}>0$ such that

$$
\begin{aligned}
& \left|f\left(t, x, y_{1}, \ldots, y_{L}\right)-f\left(t, \bar{x}, \bar{y}_{1}, \ldots, \bar{y}_{L}\right)\right| \\
& \quad \vee\left|g\left(t, x, y_{1}, \ldots, y_{L}\right)-g\left(t, \bar{x}, \bar{y}_{1}, \ldots, \bar{y}_{L}\right)\right| \\
& \quad \leq c_{j}\left(|x-\bar{x}|+\sum_{i=1}^{L}\left|y_{i}-\bar{y}_{i}\right|\right)
\end{aligned}
$$

for all $t \geq 0$ and those $x, \bar{x}, y_{i}, \bar{y}_{i} \in \mathbb{R}^{n}$ with $|x| \vee|\bar{x}| \vee\left|y_{i}\right| \vee$ $\left|\bar{y}_{i}\right| \leq j(1 \leq i \leq L)$. 
The following lemma shows the bounded property of polynomial functions.

Lemma 2. For any $h(x) \in C\left(\mathbb{R}^{n} ; \mathbb{R}_{+}\right)$and positive constants a and $q$, if $h(x)=o\left(|x|^{q}\right)$ as $|x| \rightarrow \infty$, then

$$
\sup _{x \in \mathbb{R}^{n}}\left[-a|x|^{q}+o\left(|x|^{q}\right)\right]<\infty .
$$

Proof. Choose $r>0$ such that $h(x)<a|x|^{q}$ when $x \in$ $\mathbb{R}^{n},|x|>r$, which implies that $-a|x|^{q}+o\left(|x|^{q}\right)<0$. We therefore have

$$
\begin{aligned}
\sup _{x \in \mathbb{R}^{n}} & {\left[-a|x|^{q}+o\left(|x|^{q}\right)\right] } \\
& =\sup _{x \in \mathbb{R}^{n},|x| \leq r}\left[-a|x|^{q}+o\left(|x|^{q}\right)\right]<\infty,
\end{aligned}
$$

as required.

To proceed, we need a lemma which will play a crucial role in overcoming the difficulties for the existence of unbounded delays. For the sake of simplicity, we denote

$$
\Phi_{\varepsilon}:=\Phi_{\varepsilon}(t, x, Y)=\sum_{k=1}^{K} \sum_{l=1}^{L} a_{k}\left[\left|y_{l}\right|^{\alpha_{k}} e^{-\varepsilon \bar{q}_{l} t}-q_{l}^{-1}|x|^{\alpha_{k}}\right],
$$

where $a_{k}, \alpha_{k}(1 \leq k \leq K)$ are all positive constants and $\varepsilon \geq 0$, $\bar{q}_{l}=1-q_{l}$. If $\varepsilon=0$, then we have

$$
\Phi:=\Phi(x, Y)=\sum_{k=1}^{K} \sum_{l=1}^{L} a_{k}\left[\left|y_{l}\right|^{\alpha_{k}}-q_{l}^{-1}|x|^{\alpha_{k}}\right] .
$$

Lemma 3. Assume that $0 \leq \gamma \leq \varepsilon$. If $x(t)$ is a solution to (4) with initial data $x_{0} \in \mathbb{R}^{n}$, then

$$
\int_{0}^{t} e^{\gamma s} \Phi_{\varepsilon}(s, x(s), Y(s)) d s \leq 0 .
$$

Proof. Let $I$ denote the left side of (17). We compute

$$
\begin{aligned}
I & =\sum_{k=1}^{K} \sum_{l=1}^{L} a_{k}\left[\int_{0}^{t} e^{\gamma s}\left(e^{-\varepsilon \bar{q}_{l} s}\left|y_{l}(s)\right|^{\alpha_{k}}-q_{l}^{-1}|x(s)|^{\alpha_{k}}\right) d s\right] \\
& =\sum_{k=1}^{K} \sum_{l=1}^{L} a_{k}\left[\int_{0}^{t} e^{\gamma s} e^{-\varepsilon \bar{q}_{l} s}\left|x\left(q_{l} s\right)\right|^{\alpha_{k}} d s\right. \\
& \left.-q_{l}^{-1} \int_{0}^{t} e^{\gamma s}|x(s)|^{\alpha_{k}} d s\right] \\
& \leq \sum_{k=1}^{K} \sum_{l=1}^{L} a_{k}\left[\int_{0}^{t} e^{\gamma q_{l} s}\left|x\left(q_{l} s\right)\right|^{\alpha_{k}} d s\right. \\
& \left.-q_{l}^{-1} \int_{0}^{t} e^{\gamma s}|x(s)|^{\alpha_{k}} d s\right] \\
& \leq \sum_{k=1}^{K} \sum_{l=1}^{L} a_{k}\left[q_{l}^{-1} \int_{0}^{q_{l} t} e^{\gamma s}|x(s)|^{\alpha_{k}} d s\right.
\end{aligned}
$$

$$
\begin{aligned}
& \left.-q_{l}^{-1} \int_{0}^{t} e^{\gamma s}|x(s)|^{\alpha_{k}} d s\right] \\
& =\sum_{k=1}^{K} \sum_{l=1}^{L} a_{k}\left[q_{l}^{-1} \int_{t}^{q_{l} t} e^{\gamma s}|x(s)|^{\alpha_{k}} d s\right] \leq 0 .
\end{aligned}
$$

The proof is complete.

\section{A General Theorem}

In this section, by Lyapunov function techniques, we establish a general theorem for the existence and boundedness of the global solution to (4).

Theorem 4. Assume that there exist positive constants $\varepsilon, p, a$, letting the function $V(x)=|x|^{p}$, if

$$
\begin{aligned}
\mathscr{L} V(t, x, Y)+\varepsilon V(x) \leq & \Phi_{\varepsilon}-a|x|^{q}+o\left(|x|^{q}\right), \\
& \left(t \geq 0, x \in \mathbb{R}^{n}, Y \in \mathbb{R}^{n \times L}\right),
\end{aligned}
$$

where $\Phi_{\varepsilon}$ is defined by (15) and $\mathscr{L} V(t, x, Y)$ is defined by (7). Then, for any given initial data $x_{0} \in \mathbb{R}^{n}$, there exists a unique global solution $x\left(t, x_{0}\right)(0 \leq t<\infty)$ to (4) which obeys (5) and (6).

Proof. For initial data $x_{0} \in \mathbb{R}^{n}$, the proof will be divided into three steps.

Step 1 (existence of the global solution). By Assumption 1, there exists a unique maximal local solution $x(t)=x\left(t, x_{0}\right)\left(-\tau_{0} \leq\right.$ $t<\sigma$ ) to (4), where $\sigma$ is the explosion time. Let $k_{0}$ be a sufficiently large positive number such that $\left|x_{0}\right| \leq k_{0}$. For each integer $k \geq k_{0}$, define the stopping time:

$$
\sigma_{k}=\inf \left\{-\tau_{0} \leq t<\sigma: V(x(t)) \geq k\right\} .
$$

Clearly, $\sigma_{k}$ is increasing and $\sigma_{k} \rightarrow \sigma_{\infty} \leq \sigma$ as $k \rightarrow \infty$. If we can show $\sigma_{\infty}=\infty$, a.s., then $\sigma=\infty$ a.s., which implies the desired result. This is also equivalent to proving that, for any $t>0, \mathbb{P}\left(\sigma_{k} \leq t\right) \rightarrow 0$ as $k \rightarrow \infty$. Letting $t_{k}=t \wedge \sigma_{k}$, by the Itô formula (19), and noting that $\Phi_{\varepsilon}$ is decreasing in $\varepsilon$, we have

$$
\begin{aligned}
& k \mathbb{P}\left(\sigma_{k} \leq t\right) \leq \mathbb{E} V\left(x\left(t_{k}\right)\right)=\text { const } \\
& +\mathbb{E} \int_{0}^{t_{k}} L V(x(s)) d s \leq \mathrm{const} \\
& +\mathbb{E} \int_{0}^{t_{k}}\left[\Phi_{\varepsilon}(s, x(s), Y(s))-\varepsilon V(x)-a|x(s)|^{q}\right. \\
& \left.+o\left(|x|^{q}\right)\right] d s \leq \mathrm{const}+\mathbb{E} \int_{0}^{t_{k}}[\Phi(x(s), Y(s)) \\
& +K] d s \leq \text { const }+K t,
\end{aligned}
$$

where we have used Lemmas 2 and 3. In this paper, const and $K$ always represent some positive constants whose values are not important. 
Therefore,

$$
\mathbb{P}\left(\sigma_{k} \leq t\right) \longrightarrow 0 \text { as } k \longrightarrow \infty,
$$

as required.

Step 2 (moment boundedness). Applying the Itô formula and connecting (19) with Lemmas 2 and 3, we compute

$$
\begin{aligned}
e^{\varepsilon t} \mathbb{E} V(x(t))= & \mathbb{E} V(x(0)) \\
& +\mathbb{E} \int_{0}^{t} e^{\varepsilon s}[L V(x(s))+\varepsilon V(x(s))] d s \\
\leq & \text { const } \\
& +\mathbb{E} \int_{0}^{t} e^{\varepsilon s}\left[\Phi_{\varepsilon}(s, x(s), Y(s))+K\right] d s \\
\leq & \text { const }+\varepsilon^{-1} K e^{\varepsilon t},
\end{aligned}
$$

which implies that

$$
\limsup _{t \rightarrow \infty} \mathbb{E} V(x(t)) \leq \varepsilon^{-1} K,
$$

as desired.

Step 3 (moment boundedness average in time). By (19), applying the Itô formula to $V(x)$ yields

$$
\begin{aligned}
& a \int_{0}^{t} \mathbb{E}|x(s)|^{q} d s \leq \mathbb{E} \int_{0}^{t}[-\mathscr{L} V(s, x(s), Y(s)) \\
& \quad+\Phi(x(s), Y(s))+K] d s \leq-[\mathbb{E} V(x(t)) \\
& \quad-\mathbb{E} V(x(0))]+ \text { const }+K t \leq-\mathbb{E} V(x(t))+\mathrm{const} \\
& \quad+K t \leq \mathrm{const}+\mathrm{Kt},
\end{aligned}
$$

which implies the desired (6). The proof is complete.

Remark 5. In existence of the global solution, it is not necessary to specify $V(x)=|x|^{p}$. If the function $V(x)$ satisfies $\lim \inf _{|x| \rightarrow \infty} V(x)=\infty$, existence of the global solution still holds.

After completing the proof of the general theorem, we continue to examine it in both ways. On one hand, (5) and (6) are the two main results whose understanding can be enriched as the following corollary shows.

Corollary 6. Let $x(t)$ be a positive stochastic process with properties (5) and (6). If $0<\gamma<p<\infty$ and $0<\bar{\gamma}<q<\infty$, then

$$
\begin{array}{r}
\limsup _{t \rightarrow \infty} \mathbb{E}|x(t)|^{\gamma} \leq K_{\gamma}, \\
\limsup _{t \rightarrow \infty} \frac{1}{t} \int_{0}^{t} \mathbb{E}|x(s)|^{\bar{\gamma}} d s \leq L_{\bar{\gamma}},
\end{array}
$$

where $K_{\gamma}$ and $L_{\bar{\gamma}}$ are positive constants, which may be dependent on $K_{p}, L_{q}$, and $\gamma, \bar{\gamma}$.
Proof. By the Lyapunov inequality, for any $p$ th integrable random variable $X$, we have

$$
\left(\mathbb{E}|X|^{\gamma}\right)^{1 / \gamma} \leq\left(\mathbb{E}|X|^{p}\right)^{1 / p},
$$

which gives the first result. By the Lyapunov inequality, the Hölder inequality, and (6),

$$
\begin{aligned}
& \limsup _{t \rightarrow \infty} \frac{1}{t} \int_{0}^{t} \mathbb{E}|x(s)|^{\bar{\gamma}} d s \\
& \leq \limsup _{t \rightarrow \infty} \frac{1}{t} \int_{0}^{t}\left[\mathbb{E}|x(s)|^{q}\right]^{\bar{\gamma} / q} d s \\
& \quad \leq \limsup _{t \rightarrow \infty} \frac{1}{t}\left(\int_{0}^{t} d s\right)^{(q-\bar{\gamma}) / q}\left[\int_{0}^{t} \mathbb{E}|x(s)|^{q} d s\right]^{\bar{\gamma} / q} \\
& \quad=\limsup _{t \rightarrow \infty}\left[\frac{1}{t} \int_{0}^{t} \mathbb{E}|x(s)|^{q} d s\right]^{\bar{\gamma} / q} \leq\left(L_{q}\right)^{\bar{\gamma} / q} .
\end{aligned}
$$

This completes the proof.

On the other hand, condition (19) is inconvenient to be checked because it is unrelated to functions $f$ and $g$ explicitly. To make Theorem 4 more applicable, one natural alternative is to look for other simplified conditions on $f$ and $g$. Applying (7) to $V=|x|^{p}$ leads to

$$
\begin{aligned}
\mathscr{L} V(t, x, Y)= & p|x|^{p-2} x^{T} f(t, x, Y) \\
& +\frac{p}{2}|x|^{p-2}|g(t, x, Y)|^{2} \\
& +\frac{p(p-2)}{2}|x|^{p-4}\left|x^{T} g(t, x, Y)\right|^{2} \\
:= & I_{1}+I_{2}+I_{3},
\end{aligned}
$$

where $Y \in \mathbb{R}^{n \times L}$ is defined in (11). By (19), if we can test

$$
I_{1}+I_{2}+I_{3} \leq \Phi_{\varepsilon}(t, x, Y)-a|x|^{q}+o\left(|x|^{q}\right),
$$

where $q$ and $a$ represent positive constants, $q>p, o\left(|x|^{q}\right)$ denotes some $h(x) \in C\left(\mathbb{R}^{n}\right)$ which satisfies $|x|^{-q} h(x) \rightarrow$ 0 as $|x| \rightarrow \infty$. By Lemma 2 we can easily decide that $\sup _{x \in \mathbb{R}^{n}}\left[-b|x|^{q}+o\left(|x|^{q}\right)\right]<\infty$, which implies that Theorem 4 will hold.

In the next section, we give some alternative conditions to guarantee Theorem 4 , which shows coefficients $f$ and $g$ how to determine existence of global solution to (4) and boundedness of this solution.

\section{Main Results}

To match (30), we will impose the following two groups of conditions on the functions $f$ and $g$, which shows that the growth of both $f$ and $g$ is polynomial or controlled by polynomial speed.

For any $x \in \mathbb{R}^{n}, Y \in \mathbb{R}^{n \times L}$, and $t \geq 0$,

$$
\begin{aligned}
& \left(\mathrm{A}_{1}\right) x^{T} f(t, x, Y) \leq-\sigma|x|^{\alpha+2}+\sum_{l=1}^{L} \bar{\sigma}_{l}\left|y_{l}\right|^{\alpha+2} e^{-\varepsilon \bar{q}_{l} t}+ \\
& o\left(|x|^{\alpha+2}\right),
\end{aligned}
$$


$\left(\mathrm{A}_{2}\right)|g(t, x, Y)| \leq \lambda|x|^{\beta+1}+\sum_{l=1}^{L} \bar{\lambda}_{l}\left|y_{l}\right|^{\beta+1} e^{-\varepsilon \bar{q}_{l} t}+o\left(|x|^{\beta+1}\right)$, and

$\left(\mathrm{B}_{1}\right)|x|^{-1}|f(t, x, Y)| \leq \gamma|x|^{\alpha}+\sum_{l=1}^{L} \bar{\gamma}_{l}\left|y_{l}\right|^{\alpha} e^{-\varepsilon \bar{q}_{l} t}+o\left(|x|^{\alpha}\right)$,

$\left(\mathrm{B}_{2}\right)|x|^{-1}|g(t, x, Y)| \leq \lambda|x|^{\beta}+\sum_{l=1}^{L} \bar{\lambda}_{l}\left|y_{l}\right|^{\beta} e^{-\varepsilon \bar{q}_{l} t}+o\left(|x|^{\beta}\right)$,

$\left(\mathrm{B}_{3}\right)-|x|^{-4}\left|x^{T} g(t, x, Y)\right|^{2} \leq-\sigma|x|^{2 \beta}+\sum_{l=1}^{L} \bar{\sigma}_{l}\left|y_{l}\right|^{2 \beta} e^{-\varepsilon \bar{q}_{l} t}+$ $o\left(|x|^{2 \beta}\right)$,

where all parameters are positive. Since $e^{-\varepsilon \bar{q}_{l} t}$ is decreasing in $\varepsilon$, the above conditions will still hold when $\varepsilon$ is replaced by any $\varepsilon^{\prime} \in(0, \varepsilon]$.

For the purpose of simplicity, define some notations:

$$
\begin{aligned}
& \tilde{\lambda}=\lambda+\sum_{l=1}^{L} \bar{\lambda}_{l} ; \\
& \hat{\lambda}=\lambda+\sum_{l=1}^{L} \bar{\lambda}_{l} q_{l}^{-1} ; \\
& \widehat{\sigma}=\sigma-\sum_{l=1}^{L} \bar{\sigma}_{l} q_{l}^{-1} ; \\
& \widehat{\gamma}=\gamma+\sum_{l=1}^{L} \bar{\gamma}_{l} q_{l}^{-1} .
\end{aligned}
$$

Theorem 7. Let Assumption 1 hold. Under conditions $\left(A_{1}\right)$ and $\left(A_{2}\right)$, if $\alpha \geq 2 \beta$ and

$$
2 \widehat{\sigma}>\tilde{\lambda} \hat{\lambda} H(2 \beta-\alpha),
$$

then, for any initial data $x_{0} \in \mathbb{R}^{n}$, there exists a unique global solution $x\left(t, x_{0}\right)$ to (4) which obeys (5) and (6), where $p \in$ $\left(0, p_{0}\right), q \in\left(0, \alpha+p_{0}\right) . H(x)=0$ if $x<0$, and $H(x)=1$ if $x \geq 0$, and $p_{0}$ is defined as

$$
p_{0}=1+2 \widehat{\sigma}[\widetilde{\lambda} \hat{\lambda} H(2 \beta-\alpha)]^{-1} .
$$

In particular, $p_{0}=\infty$ if $\alpha>2 \beta$.

Proof. By (33) and (34), we have $p_{0}>2$. For any $p \in\left(2, p_{0}\right)$, by conditions $\left(\mathrm{A}_{1}\right)$ and $\left(\mathrm{A}_{2}\right)$, we estimate $I_{1} \sim I_{3}$. By $\left(\mathrm{A}_{1}\right)$ and the Young inequality,

$$
\begin{aligned}
I_{1} & =p|x|^{p-2} x^{T} f(t, x, Y) \leq p|x|^{p-2} \\
& \cdot\left[-\sigma|x|^{\alpha+2}+\sum_{l=1}^{L} \bar{\sigma}_{l}\left|y_{l}\right|^{\alpha+2} e^{-\varepsilon \bar{q}_{l} t}+o\left(|x|^{\alpha+2}\right)\right] \\
& \leq-p \sigma|x|^{\alpha+p} \\
& +p \sum_{l=1}^{L} \bar{\sigma}_{l} e^{-\varepsilon \bar{q}_{l} t} \frac{(p-2)|x|^{\alpha+p}+(\alpha+2)\left|y_{l}\right|^{\alpha+p}}{\alpha+p} \\
& +o\left(|x|^{\alpha+p}\right) \leq-p \sigma|x|^{\alpha+p} \\
& +p \sum_{l=1}^{L} \bar{\sigma}_{l} \frac{(p-2)|x|^{\alpha+p}+(\alpha+2)\left|y_{l}\right|^{\alpha+p} e^{-\varepsilon \bar{q}_{l} t}}{\alpha+p} \\
& +o\left(|x|^{\alpha+p}\right) .
\end{aligned}
$$

Let $x_{i} \in \mathbb{R}$ and $\delta_{i} \geq 0$. By the Cauchy inequality, we have

$$
\left(\sum_{i=1}^{n} \delta_{i} x_{i}\right)^{2} \leq \sum_{i=1}^{n} \delta_{i} \sum_{i=1}^{n} \delta_{i} x_{i}^{2} .
$$
$\mathbb{R}$,

Recall the elementary inequality: for any $0<\rho<1, a, b \in$

$$
(a+b)^{2} \leq \frac{a^{2}}{\rho}+\frac{b^{2}}{1-\rho} .
$$

Noting that $p>2$, by $\left(\mathrm{A}_{2}\right)$, and combining the Young inequality and inequalities (36) and (37) we estimate

$$
\begin{aligned}
I_{2} & +I_{3}=\frac{p}{2}|x|^{p-2}|g(t, x, Y)|^{2}+\frac{p(p-2)}{2}|x|^{p-4} \\
& \cdot\left|x^{T} g(t, x, Y)\right|^{2} \leq \frac{p(p-1)}{2}|x|^{p-2}|g(t, x, Y)|^{2} \\
& \leq \frac{p(p-1)}{2}|x|^{p-2}\left[\lambda|x|^{\beta+1}+\sum_{l=1}^{L} \bar{\lambda}_{l}\left|y_{l}\right|^{\beta+1} e^{-\varepsilon \bar{q}_{l} t}\right. \\
& \left.+o\left(|x|^{\beta}\right)\right]^{2} \leq \frac{p(p-1)}{2 \rho}|x|^{p-2}\left[\lambda|x|^{\beta+1}\right. \\
& \left.+\sum_{l=1}^{L} \bar{\lambda}_{l}\left|y_{l}\right|^{\beta+1} e^{-\varepsilon \bar{q}_{l} t}\right]^{2}+\frac{p(p-1)}{2(1-\rho)} o\left(|x|^{2 \beta+p}\right) \\
& \leq \frac{p(p-1)}{2 \rho}|x|^{p-2} \tilde{\lambda}\left[\lambda|x|^{2 \beta+2}\right. \\
& \left.+\sum_{l=1}^{L} \bar{\lambda}_{l}\left|y_{l}\right|^{2 \beta+2} e^{-2 \varepsilon \bar{q}_{l} t}\right]+o\left(|x|^{2 \beta+p}\right) \leq \frac{p(p-1)}{2 \rho} \\
& +\tilde{\lambda}\left[\lambda|x|^{2 \beta+p}\right. \\
& \left.+\sum_{l=1}^{L} \bar{\lambda}_{l} \frac{(p-2)|x|^{2 \beta+p}+(2 \beta+2) e^{-\varepsilon \bar{q}_{l} t}\left|y_{l}\right|^{2 \beta+p}}{2 \beta+p}\right] \\
& +o\left(|x|^{2 \beta+p}\right) .
\end{aligned}
$$

We therefore have

$$
I_{1}+I_{2}+I_{3} \leq \Phi_{\varepsilon}+I,
$$

where

$$
\begin{aligned}
\Phi_{\varepsilon} & =\frac{p(\alpha+2)}{\alpha+p} \sum_{l=1}^{L} \bar{\sigma}_{l}\left[e^{-\varepsilon \bar{q}_{l} t}\left|y_{l}\right|^{\alpha+p}-q_{l}^{-1}|x|^{\alpha+p}\right] \\
& +\frac{(\beta+1) p(p-1) \tilde{\lambda}}{\rho(2 \beta+p)} \\
& \cdot \sum_{l=1}^{L} \bar{\lambda}_{l}\left[e^{-\varepsilon \bar{q}_{l} t}\left|y_{l}\right|^{2 \beta+p}-q_{l}^{-1}|x|^{2 \beta+p}\right]
\end{aligned}
$$




$$
\begin{aligned}
I= & -p \sigma|x|^{\alpha+p}+\frac{p}{\alpha+p} \\
& \cdot \sum_{l=1}^{L} \bar{\sigma}_{l}\left[p-2+(\alpha+2) q_{l}^{-1}\right]|x|^{\alpha+p}+\frac{p(p-1)}{2 \rho} \\
& \cdot \tilde{\lambda}\left[\lambda+\sum_{l=1}^{L} \bar{\lambda}_{l} \frac{(p-2)+(2 \beta+2) q_{l}^{-1}}{2 \beta+p}\right]|x|^{2 \beta+p} \\
& +o\left(|x|^{\alpha+p}\right) \leq-p \sigma|x|^{\alpha+p}+p \sum_{l=1}^{L} \bar{\sigma}_{l} q_{l}^{-1}|x|^{\alpha+p} \\
& +\frac{p(p-1)}{2 \rho} \tilde{\lambda}\left[\lambda+\sum_{l=1}^{L} \bar{\lambda}_{l} q_{l}^{-1}\right]|x|^{2 \beta+p}+o\left(|x|^{\alpha+p}\right) \\
& :=-p a(\rho)|x|^{\alpha+p}+o\left(|x|^{\alpha+p}\right),
\end{aligned}
$$

in which we have used $q_{l} \leq 1(1 \leq l \leq L)$. By (33) and (34),

$$
\begin{aligned}
a(\rho=1)= & \sigma-\sum_{l=1}^{L} \bar{\sigma} q_{l}^{-1} \\
& -\frac{(p-1) \tilde{\lambda}}{2}\left(\lambda+\sum_{l=1}^{L} \bar{\lambda}_{l} q_{l}^{-1}\right) H(2 \beta-\alpha) \\
> & \widehat{\sigma}-\frac{\left(p_{0}-1\right) \tilde{\lambda}}{2} \hat{\lambda} H(2 \beta-\alpha)=0 .
\end{aligned}
$$

Choose $\rho$ sufficiently near 1 such that $a(\rho)>0$, which shows that (39) has similar expression to (30). By Theorem 4 and Corollary 6 , there almost surely exists a unique global solution to (4), and for any $p \in\left(0, p_{0}\right)$ and $q \in\left(0, \alpha+p_{0}\right)$, this solution still holds properties (5) and (6), as required.

If $\alpha \leq 2 \beta$, for any initial data $x_{0} \in \mathbb{R}_{\times}^{n}$, we give the following lemma for existence of global solution to (4).

Lemma 8. Let Assumption 1 hold. For positive constants $p$ and $K$, letting $V(x)=|x|^{p}-p \ln |x|$, if for any $x \in \mathbb{R}_{\times}^{n}, Y \in \mathbb{R}_{\times}^{n L}$ and $t \geq 0$,

$$
\mathscr{L} V(t, x, Y) \leq \Phi(x, Y)+K
$$

where $\Phi(x, Y)$ defined by (16). Then, for any given $x_{0} \in \mathbb{R}_{\times}^{n}$, there exists a unique global solution to (4).

Proof. Similar to the proof of Theorem 4, for any initial data $x_{0} \in \mathbb{R}_{\times}^{n}$, there almost surely exists a unique maximal local solution $x(t)=x\left(t, x_{0}\right)\left(-\tau_{0} \leq t<\sigma\right)$ to (4). Similar to the stopping time (20), define the stopping time (here we still use $\left.\sigma_{k}\right)$

$$
\sigma_{k}=\inf \left\{-\tau_{0} \leq t<\sigma:|x(t)| \notin\left(k^{-1}, k\right)\right\} \text { for each integer } k .
$$

We still need to prove $\mathbb{P}\left(\sigma_{k} \leq t\right) \rightarrow 0$ as $k \rightarrow \infty$ for any $t \geq 0$. For any $y>0$, let $U(y)=y^{p}-p \ln y$ and $D_{k}:=\left\{x: k^{-1}<\right.$ $|x|<k\}$. By the definition of $\sigma_{k}$, we have $x\left(\sigma_{k}\right) \in \partial D_{k}$ when $\sigma_{k}<\infty$, which implies that

$$
V\left(x\left(\sigma_{k}\right)\right) \geq U\left(k^{-1}\right) \wedge U(k):=u(k) .
$$

We therefore have

$$
\begin{aligned}
\mathbb{P}\left(\sigma_{k} \leq t\right) u(k) \leq & \mathbb{P}\left(\sigma_{k} \leq t\right) V\left(x\left(\sigma_{k}\right)\right) \\
\leq & \mathbb{E} V\left(x\left(t \wedge \sigma_{k}\right)\right) \\
\leq & \mathbb{E} V(x(0))+\mathbb{E} \int_{0}^{t_{k}} L V(x(s)) d s \\
\leq & \mathbb{E} V(x(0)) \\
& +\mathbb{E} \int_{0}^{t_{k}}[\Phi(x(s), Y(s))+K] d s \\
\leq & \text { const }+K t .
\end{aligned}
$$

Note that $u(k) \rightarrow \infty$ as $k \rightarrow \infty$. Letting $k \rightarrow \infty$, for any $t \geq 0$, we have

$$
\mathbb{P}\left(\sigma_{k} \leq t\right) \longrightarrow 0, \text { as } k \longrightarrow \infty
$$

as required.

Applying this lemma, we may obtain the following theorem.

Theorem 9. Let Assumption 1 hold. Under conditions ( $\left.B_{1}\right)$, $\left(B_{2}\right)$, and $\left(B_{3}\right)$, if $\alpha \leq 2 \beta$ and

$$
2 \widehat{\sigma}>\tilde{\lambda} \widehat{\lambda}+2 \widehat{\gamma} H(\alpha-2 \beta),
$$

then, for any initial data $x_{0} \in \mathbb{R}_{\times}^{n}$, there exists a unique global solution to (4) and this solution has properties (5) and (6) in which $0<p<p_{0}$ and $0<q<2 \beta+p_{0}$ and $p_{0}$ is defined as

$$
p_{0}=2-\widehat{\sigma}^{-1}[\tilde{\lambda} \hat{\lambda}+2 \widehat{\gamma} H(\alpha-2 \beta)] \text {. }
$$

Proof. By (48), $p_{0} \in(0,2)$. Fix any $p \in\left(0, p_{0}\right)$; using $\left(\mathrm{B}_{1}\right)$, $\left(\mathrm{B}_{2}\right)$, and $\left(\mathrm{B}_{3}\right)$, we estimate $I_{1} \sim I_{3}$ respectively. By the Young inequality and $\left(B_{1}\right)$, we have

$$
\begin{aligned}
I_{1} & =p|x|^{p-2} x^{T} f(t, x, Y) \\
\leq & p|x|^{p}\left[\gamma|x|^{\alpha}+\sum_{l=1}^{L} \bar{\gamma}_{l}\left|y_{l}\right|^{\alpha} e^{-\varepsilon \bar{q}_{l} t}+o\left(|x|^{\alpha}\right)\right] \\
\leq & p \gamma|x|^{\alpha+p}+p \sum_{l=1}^{L} \bar{\gamma}_{l} e^{-\varepsilon \bar{q}_{l} t} \frac{p|x|^{\alpha+p}+\alpha\left|y_{l}\right|^{\alpha+p}}{\alpha+p} \\
& +o\left(|x|^{\alpha+p}\right) \\
& \leq p \gamma|x|^{\alpha+p}+p \sum_{l=1}^{L} \bar{\gamma}_{l} \frac{p|x|^{\alpha+p}+\alpha\left|y_{l}\right|^{\alpha+p} e^{-\varepsilon \bar{q}_{l} t}}{\alpha+p} \\
& +o\left(|x|^{\alpha+p}\right) .
\end{aligned}
$$


By $\left(\mathrm{B}_{2}\right)$ and elementary inequalities (36) and (37), for any $\rho \in$ $(0,1)$, we have

$$
\begin{aligned}
I_{2} & =\frac{p}{2}|x|^{p-2}|g(t, x, Y)|^{2} \leq \frac{p}{2}|x|^{p} \\
& \cdot\left[\lambda|x|^{\beta}+\sum_{l=1}^{L} \bar{\lambda}_{l} e^{-\varepsilon \bar{q}_{l} t}\left|y_{l}\right|^{\beta}+o\left(|x|^{\beta}\right)\right]^{2} \leq \frac{p}{2 \rho}|x|^{p} \\
& \cdot\left[\lambda|x|^{\beta}+\sum_{l=1}^{L} \bar{\lambda}_{l} e^{-\varepsilon \bar{q}_{l} t}\left|y_{l}\right|^{\beta}\right]^{2}+o\left(|x|^{2 \beta+p}\right) \\
& \leq \frac{p}{2 \rho}|x|^{p} \tilde{\lambda}\left[\lambda|x|^{2 \beta}+\sum_{l=1}^{L} \bar{\lambda}_{l} e^{-2 \varepsilon \bar{q}_{l} t}\left|y_{l}\right|^{2 \beta}\right] \\
& +o\left(|x|^{2 \beta+p}\right) \leq \frac{p}{2 \rho} \\
& \cdot \tilde{\lambda}\left[\lambda|x|^{2 \beta+p}+\sum_{l=1}^{L} \bar{\lambda}_{l} e^{-\varepsilon \bar{q}_{l} t} \frac{p|x|^{2 \beta+p}+2 \beta\left|y_{l}\right|^{2 \beta+p}}{2 \beta+p}\right] \\
& +o\left(|x|^{2 \beta+p}\right) \leq \frac{p \tilde{\lambda} \lambda}{2 \rho}|x|^{2 \beta+p}+\frac{p \tilde{\lambda}}{2 \rho} \\
& +\sum_{l=1}^{L} \bar{\lambda}_{l} \frac{p|x|^{2 \beta+p}+2 \beta\left|y_{l}\right|^{2 \beta+p} e^{-\varepsilon \bar{q}_{l} t}}{2 \beta+p}+o\left(|x|^{2 \beta+p}\right) .
\end{aligned}
$$

Noting that $p<2$, by the Young inequality and condition $\left(B_{3}\right)$, we have the following estimate:

$$
\begin{aligned}
I_{3} & =\frac{p(p-2)}{2}|x|^{p-4}\left|x^{T} g(t, x, Y)\right|^{2} \leq \frac{p(p-2)}{2}|x|^{p} \\
& \cdot\left[\sigma|x|^{2 \beta}-\sum_{l=1}^{L} \bar{\sigma}_{l} e^{-\varepsilon \bar{q}_{l} t}\left|y_{l}\right|^{2 \beta}+o\left(|x|^{2 \beta}\right)\right] \\
& \leq \frac{p(p-2)}{2}\left[\sigma|x|^{2 \beta+p}\right. \\
& \left.-\sum_{l=1}^{L} \bar{\sigma}_{l} \frac{p|x|^{2 \beta+p}+2 \beta\left|y_{l}\right|^{2 \beta+p} e^{-\varepsilon \bar{q}_{l} t}}{2 \beta+p}\right]+o\left(|x|^{2 \beta+p}\right) .
\end{aligned}
$$

We therefore have

$$
I_{1}+I_{2}+I_{3} \leq \Phi_{\varepsilon}+I
$$

where

$$
\begin{aligned}
& \Phi_{\varepsilon} \\
& =\frac{\alpha p}{\alpha+p} \sum_{l=1}^{L} \bar{\gamma}_{l}\left[e^{-\varepsilon \bar{q}_{l} t}\left|y_{l}\right|^{\alpha+p}-q_{l}^{-1}|x|^{\alpha+p}\right] \\
& \quad+\frac{p \tilde{\lambda} \beta}{\rho(2 \beta+p)} \sum_{l=1}^{L} \bar{\lambda}_{l}\left[e^{-\varepsilon \bar{q}_{l} t}\left|y_{l}\right|^{2 \beta+p}-q_{l}^{-1}|x|^{2 \beta+p}\right] \\
& \quad-\frac{p(p-2) \beta}{2 \beta+p} \sum_{l=1}^{L} \bar{\sigma}_{l}\left[e^{-\varepsilon \bar{q}_{l} t}\left|y_{l}\right|^{2 \beta+p}-q_{l}^{-1}|x|^{2 \beta+p}\right],
\end{aligned}
$$

$I$

$$
\begin{aligned}
= & p \gamma|x|^{\alpha+p}+p \sum_{l=1}^{L} \bar{\gamma}_{l}\left(\frac{p+\alpha q_{l}^{-1}}{\alpha+p}\right)|x|^{\alpha+p} \\
& +\frac{p \tilde{\lambda} \lambda}{2 \rho}|x|^{2 \beta+p}+o\left(|x|^{2 \beta+p}\right) \\
& +\frac{p \tilde{\lambda}}{2 \rho} \sum_{l=1}^{L} \bar{\lambda}_{l}\left(\frac{p+2 \beta q_{l}^{-1}}{2 \beta+p}\right)|x|^{2 \beta+p} \\
& +\frac{p(p-2)}{2}\left(\sigma-\sum_{l=1}^{L} \bar{\sigma}_{l} \frac{p+2 \beta q_{l}^{-1}}{2 \beta+p}\right)|x|^{2 \beta+p} \\
\leq & \left(p \gamma+p \sum_{l=1}^{L} \bar{\gamma}_{l} q_{l}^{-1}\right)|x|^{\alpha+p} \\
& +\frac{p \tilde{\lambda}}{2 \rho}\left(\lambda+\sum_{l=1}^{L} \bar{\lambda}_{l} q_{l}^{-1}\right)|x|^{2 \beta+p} \\
& +\frac{p(p-2)}{2}\left(\sigma-\sum_{l=1}^{L} \bar{\sigma}_{l} q_{l}^{-1}\right)|x|^{2 \beta+p} \\
& +o(\rho)|x|^{2 \beta+p}+o\left(|x|^{2 \beta+p}\right),
\end{aligned}
$$

where we have used $q_{l} \leq 1(1 \leq l \leq L)$. By conditions (47) and (48) we have

$$
\begin{aligned}
\frac{2}{p} a(\rho=1)= & -2\left(\gamma+\sum_{l=1}^{L} \bar{\gamma}_{l} q_{l}^{-1}\right) H(\alpha-2 \beta) \\
& -\tilde{\lambda}\left(\lambda+\sum_{l=1}^{L} \bar{\lambda}_{l} q_{l}^{-1}\right) \\
& -(p-2)\left(\sigma-\sum_{l=1}^{L} \bar{\sigma}_{l} q_{l}^{-1}\right) \\
= & -2 \widehat{\gamma} H(\alpha-2 \beta)-\tilde{\lambda} \hat{\lambda}-(p-2) \widehat{\sigma} \\
> & -2 \widehat{\gamma} H(\alpha-2 \beta)-\tilde{\lambda} \hat{\lambda}-\left(p_{0}-2\right) \widehat{\sigma}=0 .
\end{aligned}
$$

Choose $\rho$ sufficiently near 1 such that $a(\rho)>0$, which shows that (52) has similar expression to (30). By the similar proof process to Theorem 7 , we may obtain the desired result. This completes this proof.

Theorem 7 shows that the drift coefficient $f$ makes a dominant role when $\alpha \geq 2 \beta$; in particular, $g$ only needs to satisfy condition $\left(\mathrm{A}_{2}\right)$ when $\alpha>2 \beta$, while in Theorem 9 the diffusion coefficient $g$ is dominant. That is, we depend on the environmental noise to suppress the explosion of solutions and guarantee the boundedness. However, we obtained that the order of moment is lower than Theorem 7. 


\section{Examples}

In this section, we investigate the moment boundedness of two 1-dimensional stochastic multipantograph equations.

\section{Example 1. Consider}

$$
\begin{aligned}
& d x(t)=\left[-4 x^{5}(t)+u_{1}(t) x^{2}(t) x^{3}(0.25 t)\right. \\
& \left.\quad+x^{4}(t) x(0.5 t) u_{2}(t)+x^{2}(t)\right] d t+\left[x^{2}(t)\right. \\
& +u_{1}(t) x(t) x(0.25 t)+x(t) x(0.5 t) u_{2}(t) \\
& \quad+x(t)] d w(t)
\end{aligned}
$$

with initial data $x(0)=x_{0} \in \mathbb{R}, t \geq 0$, where $u_{1}(t)=e^{-0.75 \varepsilon t}$, $u_{2}(t)=e^{-0.5 \varepsilon t}, \varepsilon>0$.

Define $y_{1}(t)=x(0.25 t), y_{2}(t)=x(0.5 t)$, and

$$
\begin{aligned}
& f(t, x, y)=-4 x^{5}+e^{-0.75 \varepsilon t} x^{2} y_{1}^{3}+e^{-0.5 \varepsilon t} x^{4} y_{2}+x^{2}, \\
& g(t, x, y)=x^{2}+e^{-0.75 \varepsilon t} x y_{1}+e^{-0.5 \varepsilon t} x y_{2}+x
\end{aligned}
$$

Obviously, $f$ and $g$ satisfy the local Lipschitz condition. Then by the Young inequality we estimate

$$
\begin{aligned}
x f(t, x, y)= & -4 x^{6}+e^{-0.75 \varepsilon t} x^{3} y_{1}^{3}+e^{-0.5 \varepsilon t} x^{5} y_{2}+x^{3} \\
\leq & -4 x^{6}+e^{-0.75 \varepsilon t} \frac{x^{6}+y_{1}^{6}}{2} \\
& +e^{-0.5 \varepsilon t} \frac{5 x^{6}+y_{2}^{6}}{6}+x^{3} \\
\leq & -\frac{8}{3} x^{6}+\frac{1}{2} e^{-0.75 \varepsilon t} y_{1}^{6}+\frac{1}{6} e^{-0.5 \varepsilon t} y_{2}^{6}+x^{3}
\end{aligned}
$$

which shows that condition $\left(\mathrm{A}_{1}\right)$ will hold if $\alpha=4, \sigma=$ $8 / 3, \bar{\sigma}_{1}=1 / 2, \bar{\sigma}_{2}=1 / 6$. Obviously, we have

$$
\begin{aligned}
|g(t, x, y)|= & \left|x^{2}+e^{-0.75 \varepsilon t} x y_{1}+e^{-0.5 \varepsilon t} x y_{2}+x\right| \\
\leq & x^{2}+\frac{1}{2} e^{-0.75 \varepsilon t}\left(x^{2}+y_{1}^{2}\right) \\
& +\frac{1}{2} e^{-0.5 \varepsilon t}\left(x^{2}+y_{2}^{2}\right)+|x| \\
\leq & 2 x^{2}+\frac{1}{2} e^{-0.75 \varepsilon t} y_{1}^{2}+\frac{1}{2} e^{-0.5 \varepsilon t} y_{2}^{2}+|x|,
\end{aligned}
$$

which shows that condition $\left(\mathrm{A}_{2}\right)$ will hold if $\beta=1, \lambda=2$, $\bar{\lambda}_{1}=1 / 2$, and $\bar{\lambda}_{2}=1 / 2$.

Substituting the above parameters into (32) gives $\widehat{\sigma}=$ $1 / 3>0$. Noting that $\alpha>2 \beta$, applying Theorem 7 , we have the following result.

For any initial data $x_{0} \in \mathbb{R},(55)$ admits a unique global solution $x\left(t, x_{0}\right)$ which has the properties (5) and (6) for any $p, q \in \mathbb{R}_{+}$.
Example 2. Consider

$$
\begin{aligned}
& d x(t)=\left[-x^{3}(t)+u_{1}(t) x^{2}(t) x(0.25 t)\right. \\
& \left.\quad+u_{2}(t) x(t) x^{2}(0.5 t)+x^{2}(t)\right] d t+\left[20 x^{3}(t)\right. \\
& \quad+u_{1}(t) x^{2}(t) x(0.25 t)+u_{2}(t) x^{2}(t) x(0.5 t) \\
& \quad+x(t)] d w(t)
\end{aligned}
$$

with initial data $x(0)=x_{0} \in \mathbb{R}_{\times}, t \geq 0$, where $u_{1}(t), u_{2}(t)$, $y_{1}$, and $y_{2}$ are defined the same as in Example 1 .

Define

$$
\begin{aligned}
& f(t, x, y)=-x^{3}+e^{-0.75 \varepsilon t} x^{2} y_{1}+e^{-0.5 \varepsilon t} x y_{2}^{2}+x^{2} \\
& g(t, x, y)=20 x^{3}+e^{-0.75 \varepsilon t} x^{2} y_{1}+e^{-0.5 \varepsilon t} x^{2} y_{2}+x
\end{aligned}
$$

Obviously, $f$ and $g$ satisfy the local Lipschitz condition. By the Young inequality, we compute

$$
\begin{aligned}
|x|^{-1} & |f(t, x, y)| \\
& =|x|^{-1}\left|-x^{3}+e^{-0.75 \varepsilon t} x^{2} y_{1}+e^{-0.5 \varepsilon t} x y_{2}^{2}+x^{2}\right| \\
& \leq x^{2}+e^{-0.75 \varepsilon t} \frac{x^{2}+y_{1}^{2}}{2}+e^{-0.5 \varepsilon t} y_{2}^{2}+|x| \\
& \leq \frac{3}{2} x^{2}+\frac{1}{2} e^{-0.75 \varepsilon t} y_{1}^{2}+e^{-0.5 \varepsilon t} y_{2}^{2}+|x|,
\end{aligned}
$$

which shows that condition $\left(\mathrm{B}_{1}\right)$ will hold if $\alpha=2, \gamma=3 / 2$, $\bar{\gamma}_{1}=1 / 2$, and $\bar{\gamma}_{2}=1$. Applying the Young inequality again, we have

$$
\begin{aligned}
|x|^{-1}|g(t, x, y)| & \\
& =|x|^{-1}\left|20 x^{3}+e^{-0.75 \varepsilon t} x^{2} y_{1}+e^{-0.5 \varepsilon t} x^{2} y_{2}+x\right| \\
& \leq 20 x^{2}+e^{-0.75 \varepsilon t} \frac{x^{2}+y_{1}^{2}}{2}+e^{-0.5 \varepsilon t} \frac{x^{2}+y_{2}^{2}}{2}+1 \\
& \leq 21 x^{2}+\frac{1}{2} e^{-0.75 \varepsilon t} y_{1}^{2}+\frac{1}{2} e^{-0.5 s t} y_{2}^{2}+1,
\end{aligned}
$$

which shows that condition $\left(\mathrm{B}_{2}\right)$ will hold if $\beta=2, \lambda=21$, $\bar{\lambda}_{1}=1 / 2$, and $\bar{\lambda}_{2}=1 / 2$. $\mathbb{R}$,

Consider the elementary inequality: for any $\rho>1, a, b \in$

$$
(a+b)^{2} \geq \frac{a^{2}}{\rho}-\frac{b^{2}}{\rho-1},
$$

and recall inequality (37): for any $\rho>1,0<\delta<1$, we estimate

$$
\begin{aligned}
& x^{-2}|g(t, x, y)|^{2} \\
& =\left|20 x^{2}+e^{-0.75 \varepsilon t} x y_{1}+e^{-0.5 \varepsilon t} x y_{2}+1\right|^{2} \\
& \geq \frac{20^{2} x^{4}}{\rho}-\frac{\left[e^{-0.75 \varepsilon t} x y_{1}+e^{-0.5 \varepsilon t} x y_{2}+1\right]^{2}}{\rho-1}
\end{aligned}
$$




$$
\begin{aligned}
\geq & \frac{20^{2} x^{4}}{\rho} \\
& -\frac{1}{\rho-1}\left[\frac{\left(e^{-0.75 \varepsilon t} x y_{1}+e^{-0.5 \varepsilon t} x y_{2}\right)^{2}}{\delta}+\frac{1}{1-\delta}\right] \\
\geq & \frac{20^{2} x^{4}}{\rho}-\frac{2}{\delta(\rho-1)}\left[e^{-0.75 \varepsilon t} x^{2} y_{1}^{2}+e^{-0.5 \varepsilon t} x^{2} y_{2}^{2}\right] \\
& -\frac{1}{(\rho-1)(1-\delta)} \\
\geq & \frac{20^{2} x^{4}}{\rho} e^{-0.5 \varepsilon t} y_{2}^{4}-\frac{1}{(\rho-1)(1-\delta)} . \\
& -\frac{1}{(\rho-1)(1-\delta)}\left[e^{-0.75 \varepsilon t}\left(x^{4}+y_{1}^{4}\right)+e^{-0.5 \varepsilon t}\left(x^{4}+y_{2}^{4}\right)\right] \\
\geq & \left.\frac{20^{2}}{\rho}-\frac{1}{\delta(\rho-1)}\right] x^{4}-\frac{1}{\delta(\rho-1)} e^{-0.75 \varepsilon t} y_{1}^{4}
\end{aligned}
$$

Therefore, condition $\left(B_{3}\right)$ is satisfied with $\beta=2$ and

$$
\begin{gathered}
\sigma=\frac{20^{2}}{\rho}-\frac{2}{\delta(\rho-1)}, \\
\bar{\sigma}_{1}=\bar{\sigma}_{2}=\frac{1}{\delta(\rho-1)} .
\end{gathered}
$$

Substituting the above parameters into (31) and (32) gives $\tilde{\lambda}=$ 22, $\hat{\lambda}=24$, and

$$
\widehat{\sigma}=\frac{20^{2}}{\rho}-\frac{8}{\delta(\rho-1)} .
$$

Let $\delta \rightarrow 1$, maximizing $\widehat{\sigma}$ by choosing $\rho=20 /(20-\sqrt{8})>1$, yield

$$
\widehat{\sigma}=(20-\sqrt{8})^{2} .
$$

Noting that $\alpha<2 \beta$, then we have $2 \widehat{\sigma}=2(20-\sqrt{8})^{2}>\tilde{\lambda} \hat{\lambda}=$ 528; that is, condition (47) is satisfied and $P_{0}=2-\widehat{\sigma}^{-1} \tilde{\lambda} \hat{\lambda}=$ 0.3 .

Applying Theorem 9, we have the following result.

For any initial data $x_{0} \in \mathbb{R}_{\times},(59)$ admits a unique global solution $x\left(t, x_{0}\right)$ which has properties (5) and (6) in which $p \in(0,0.3)$ and $q \in(0,4.3)$.

\section{Further Remarks}

This paper is devoted to the asymptotic properties of the stochastic multipantograph equations. We investigate the existence and uniqueness of the global solution and its moment boundedness. Besides obtaining a general theorem, we obtain two sufficient criteria which can be much more easily verifiable than the general theorem. Two examples demonstrate our results.

Since (3) arises in the analysis of the dynamics of an overhead current collection system for an electric locomotive and applied to engineering and applied mathematics fields, the asymptotic behavior of stochastic multipantograph equation has meaningful interpretations (cf. $[4,26])$. The main idea and the method developed in this paper have the potential to investigate some other unbounded delay stochastic systems, such as neural networks, infinite-delay Kolmogorov-type systems, and Volterra equations in mathematical biology.

\section{Conflict of Interests}

The authors declare that there is no conflict of interests regarding the publication of this paper.

\section{Acknowledgments}

This research is supported by the National Social Science Foundation of China (Grant no. 14CTJ008) and the China Postdoctoral Science Foundation (Grant no. 2015M582317).

\section{References}

[1] J. M. Cushing, Integrodifferential Equations and Delay Models in Population Dynamics, Academic Press, Boston, Mass, USA, 1993.

[2] Y. Kuang, Delay Differential Equations with Applications in Population Dynamics, Academic Press, Boston, Mass, USA, 1993.

[3] J. D. Murray, Mathematical Biology, Springer, Berlin, Germany, 1993.

[4] J. K. Ockendon and A. B. Tayler, "The dynamics of a current collection system for an electric locomotive," Proceedings of the Royal Society of London Series A: Mathematical, Physical and Engineering Sciences, vol. 322, no. 1551, pp. 447-468, 1971.

[5] W. G. Ajello, H. I. Freedman, and J. Wu, "A model of stage structured population growth with density depended time delay," SIAM Journal on Applied Mathematics, vol. 521, pp. 855869, 1992.

[6] M. Buhmann and A. Iserles, "Stability of the discretized pantograph differential equation," Mathematics of Computation, vol. 60, no. 202, pp. 575-589, 1993.

[7] M. Dehghan and F. Shakeri, "The use of the decomposition procedure of Adomian for solving a delay differential equation arising in electrodynamics," Physica Scripta, vol. 78, no. 6, Article ID 065004, 2008.

[8] F. Shakeri and M. Dehghan, "Solution of delay differential equations via a homotopy perturbation method," Mathematical and Computer Modelling, vol. 48, no. 3-4, pp. 486-498, 2008.

[9] Z. C. Fan, M. Z. Liu, and W. R. Cao, "Existence and uniqueness of the solutions and convergence of semi-implicit Euler methods for stochastic pantograph equations," Journal of Mathematical Analysis and Applications, vol. 325, no. 2, pp. 1142-1159, 2007.

[10] J. A. D. Appleby and E. Buckwar, "Sufficient condition for polynomial asymptotic behaviour of stochastic pantograph equations," http://www.dcu.ie/maths/research/preprint.shtml. 
[11] C. T. H. Baker and E. Buckwar, "Continuous $\theta$-methods for the stochastic pantograph equation," Electronic Transactions on Numerical Analysis, vol. 11, pp. 131-151, 2000.

[12] Z. Fan, M. Song, and M. Liu, "The $\alpha$ th moment stability for the stochastic pantograph equation," Journal of Computational and Applied Mathematics, vol. 233, no. 2, pp. 109-120, 2009.

[13] L. Ronghua, L. Min, and P. Wan-kai, "Convergence of numerical solutions to stochastic pantograph equations with Markovian switching," Applied Mathematics and Computation, vol. 215, no. 1, pp. 414-422, 2009.

[14] X. Q. Lü and M. G. Cui, "Analytic solutions to a class of nonlinear infinite-delay-differential equations," Journal of Mathematical Analysis and Applications, vol. 343, no. 2, pp. 724-732, 2008.

[15] A. Iserles, "On the generalized pantograph functional-differential equation," European Journal of Applied Mathematics, vol. 4, no. 1, pp. 1-38, 1993.

[16] A. Iserles, "Exact and discretized stability of the pantograph equation," Applied Numerical Mathematics, vol. 24, no. 2-3, pp. 295-308, 1997.

[17] M. Z. Liu, Z. W. Yang, and G. D. Hu, "Asymptotical stability of numerical methods with constant stepsize for pantograph equations," BIT Numerical Mathematics, vol. 45, no. 4, pp. 743-759, 2005.

[18] D. Li and M. Z. Liu, "Runge-Kutta methods for the multi-pantograph delay equation," Applied Mathematics and Computation, vol. 163, no. 1, pp. 383-395, 2005.

[19] M. Z. Liu and D. S. Li, "Properties of analytic solution and numerical solution of multi-pantograph equation," Applied Mathematics and Computation, vol. 155, no. 3, pp. 853-871, 2004.

[20] Z.-H. Yu, "Variational iteration method for solving the multipantograph delay equation," Physics Letters A, vol. 372, no. 43, pp. 6475-6479, 2008.

[21] S. G. Hu, C. M. Huang, and F. K. Wu, Stochastic Differential Equation, Science Publishing, Beijing, China, 2008.

[22] X. R. Mao, Exponential Stability of Stochastic Differential Equations, Marcel Dekker, 1994.

[23] X. R. Mao, Stochastic Differential Equations and Applications, Horwood Publishing Limited, Chichester, UK, 1997.

[24] L. Arnold, Stochastic Differential Equations: Theory and Applications, John Wiley \& Sons, New York, NY, USA, 1974.

[25] S. Fang and T. Zhang, "A study of a class of stochastic differential equations with non-Lipschitzian coefficients," Probability Theory and Related Fields, vol. 132, no. 3, pp. 356-390, 2005.

[26] L. Fox, D. F. Mayers, J. R. Ockendon, and A. B. Tayler, "On a functional differential equation," Journal of the Institute of Mathematics and Its Applications, vol. 8, pp. 271-307, 1971. 


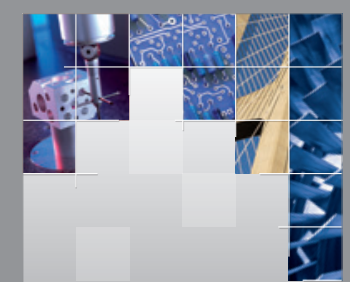

\section{Enfincering}
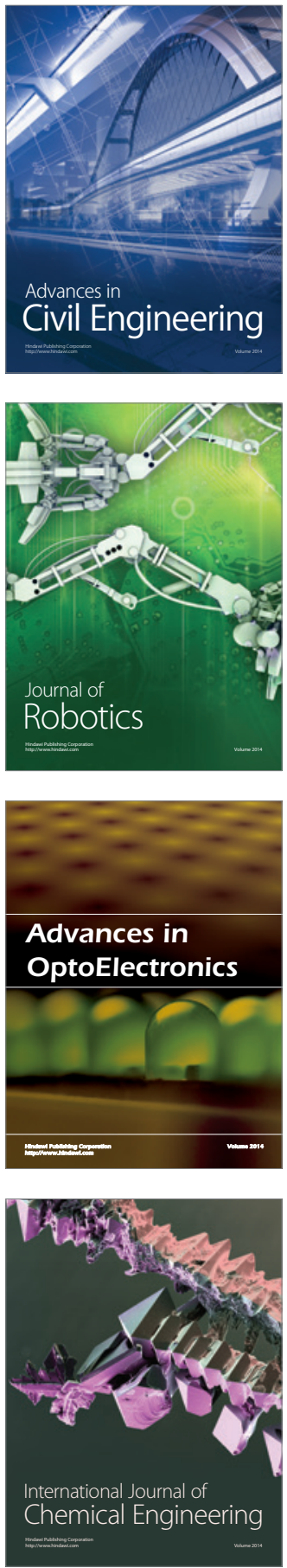

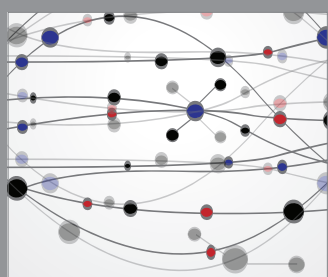

The Scientific World Journal

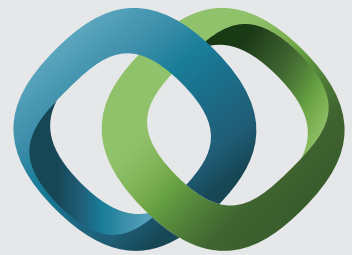

\section{Hindawi}

Submit your manuscripts at

http://www.hindawi.com
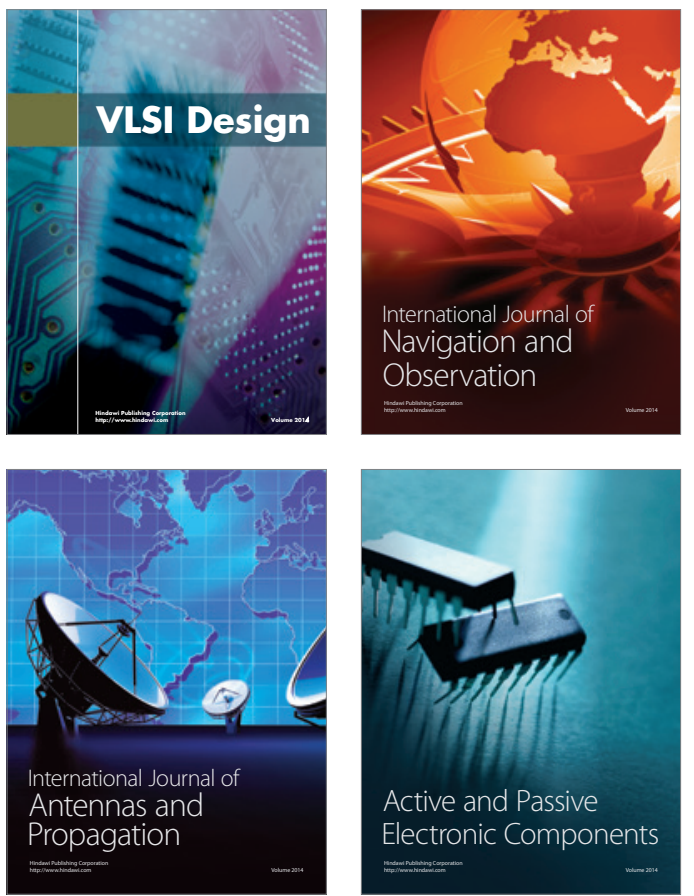
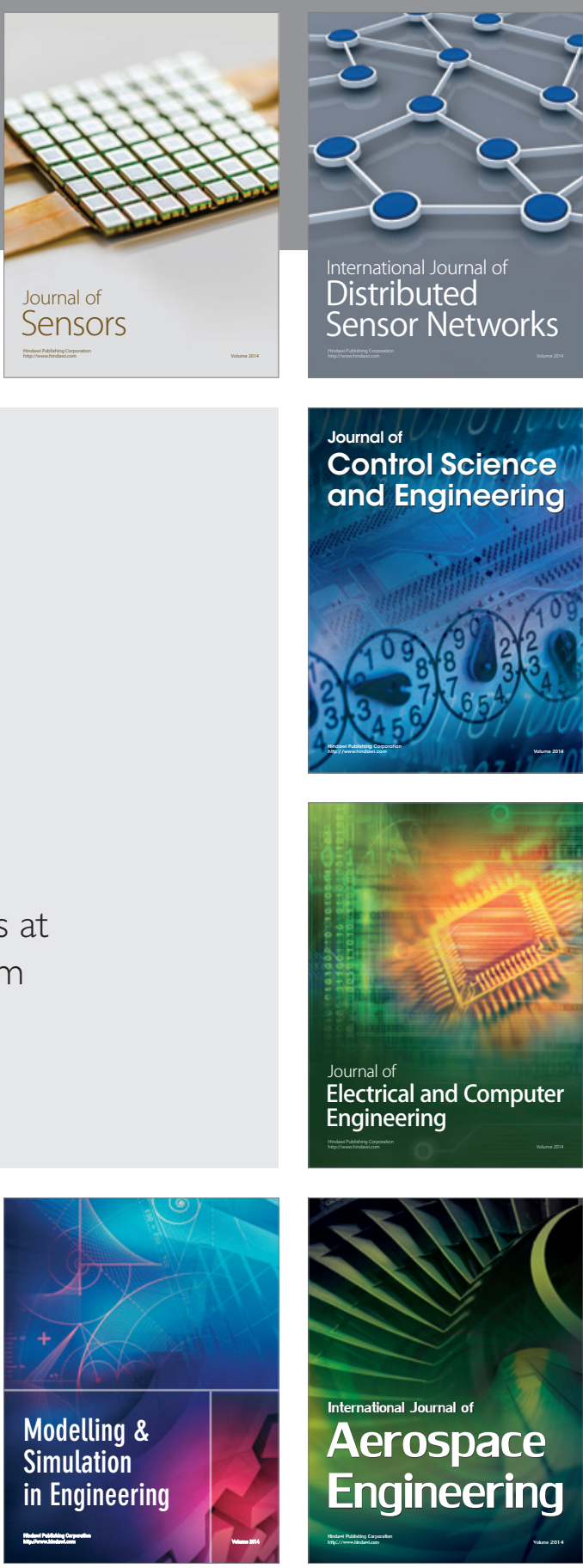

International Journal of

Distributed

Sensor Networks

Journal of

Control Science

and Engineering
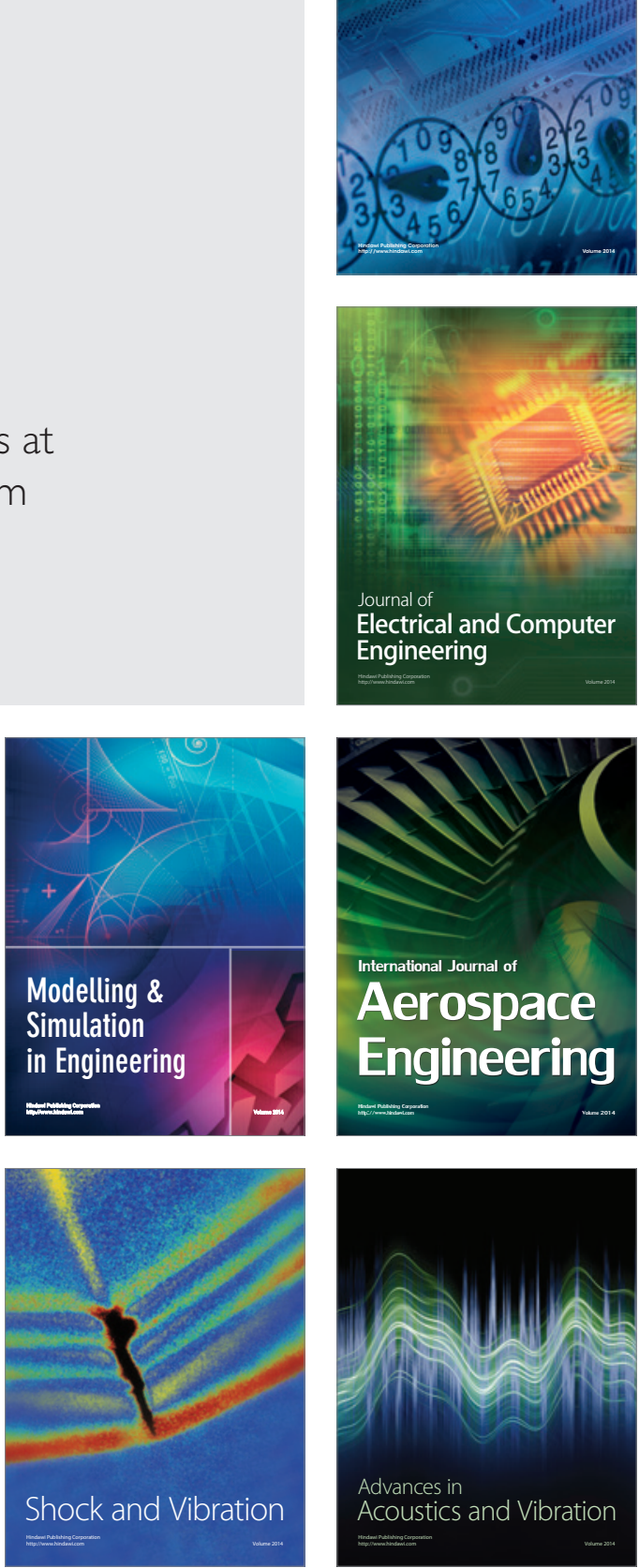